\title{
GFP Chimeras of E-MAP-115 (ensconsin) Domains Mimic Behavior of the Endogenous Protein in vitro and in vivo
}

\author{
J. Chloë Bulinski ${ }^{2}$, , Dorota Gruber ${ }^{1}$, Kathleen Faire ${ }^{1}$, Pallavi Prasad ${ }^{1}$, and Winston Chang ${ }^{2}$ \\ ${ }^{1}$ Department of Anatomy \& Cell Biology and Pathology and 2 Integrated Program in Cell, Molecular, \& Bio- \\ physical Studies, Columbia University, College of Physicians \& Surgeons, $630 \mathrm{~W}$. 168th St. New York, NY \\ 10032-3702, USA
}

\begin{abstract}
E-MAP-115 (ensconsin) is a microtubule-associated protein (MAP) abundant in carcinoma and other epithelia-derived cells. We expressed chimeras of green fluorescent protein (GFP) conjugated to ensconsin's N-terminal MT-binding domain (EMTB), to study distribution, dynamics, and function of the MAP in living cells. We tested the hypothesis that behavior of expressed GFP-EMTB accurately matched behavior of endogenous ensconsin. Like endogenous MAP, GFP-EMTB was associated with microtubules in living or fixed cells, and microtubule association of either molecule was impervious to extraction with nonionic detergents. In cell lysates both GFP-EMTB and endogenous ensconsin were dissociated from microtubules by identical salt extraction conditions, and both molecules remained bound to a calcium-stable subset of Taxolstabilized microtubules. These data show that microtubule association of ensconsin was affected neither by the absence of domains other than its microtubule-binding domain, nor by the presence of appended GFP. We took advantage of this finding to generate constructs in which additional GFP moieties were attached to EMTB, to obtain a more intensely fluorescent reporter of in vivo MAP binding. We show here that expression of chimeric proteins consisting of five GFP molecules attached to a single EMTB molecule produces brightly labeled microtubules without compromising the behavior of the MAP or the microtubules to which it is attached. Thus, we have demonstrated the utility of chimeric proteins containing GFP multimers as authentic reporters of ensconsin distribution and dynamics; expression of these GFP-EMTB chimeric molecules also provides a non-perturbing label of the microtubule system in living cells.
\end{abstract}

Key words: Multimeric GFP/Taxol/cytoskeleton/carcinoma cells/in vivo microtubule imaging

\section{Introduction}

Proteins that bind to microtubules (MTs) but not to tubulin dimers, called MT-associated proteins (MAPs), are thought to modulate the functions of MTs in a cell-type specific manner (see review by Mandelkow and Mandekow (14). In carcinoma and other epitheliaderived cells, an abundant microtubule-associated protein (MAP) is E-MAP-115 (ensconsin), a $115 \mathrm{kDa}$ basic MAP containing an N-terminal microtubule-binding domain, whose cloning and molecular characteriza-

\footnotetext{
* To whom correspondence should be addressed: Department of Anatomy \& Cell Biology-BB1213, Columbia University, College of Physicians \& Surgeons 630 W. 168th St. New York, NY 10032-3702, USA.

Tel: $+1-212-305-5113$ or $305-4183$, Fax: $+1-212-305-1413$ or 305-3970

E-mail: jcb4@columbia.edu
}

tion were reported by Masson and Kreis (15). Sequence analysis and expression cloning recently demonstrated the identity of E-MAP-115 with ensconsin, a MAP originally isolated as a major component of $\mathrm{HeLa}$ cell MT and MAP preparations $(1,21)$, and subsequently shown to bind to MTs in vitro and to stimulate MT assembly (2).

Several intriguing properties of E-MAP-115 (ensconsin) provided a compelling rationale for examining the in vivo behavior of this MAP. For example, in vitro studies demonstrated ensconsin's ability to associate more tightly with MTs upon addition of the MT-stabilizing drug, Taxol (3). In addition, the MAP was shown to undergo cell cycle associated phosphorylation changes that might affect its MT association and/or its function at the onset of M-phase (16). Expression studies also demonstrated that E-MAP-115 (ensconsin) was increased in abundance early in epithe- 
lial development in embryonic mice (9), and was correlated with differentiation of keratinocytes (10), thus raising the possibility that the MAP plays a tissue-specific role in stabilizing or functionally modifying MTs during differentiation or maintenance of epithelia. More recently, the level of expression of E-MAP-115 (ensconsin) was shown to be correlated with the degree of Taxol-sensitivity of various cultured cell lines (12), further warranting studies of its associative behavior with MTs in vivo, in the absence and presence of drugs such as Taxol.

In this short report, we demonstrate that we have established cell lines in which we have expressed GFP-chimeric proteins that faithfully monitor the distribution and MT associative properties of endogenous E-MAP115 (ensconsin). In addition to conventional GFP-chimeric proteins, we also show that addition of tandem multimers of GFP to the chimeric proteins generates a more intense fluorescent signal from the expressed protein, without compromising function of the MAP or the cells that express it. This system is likely to be amenable to the study not only of this MAP, but also of a multitude of MT-dependent processes in real time.

\section{Materials and Methods}

\section{Materials}

Unless otherwise stated, all chemicals were from Sigma (St. Louis, MO, USA) or Fisher Scientific (Tustin, CA, USA). Tissue culture materials, solutions and oligonucleotides were from GIBCO Life Sciences (Gaithersburg, MD, USA), while restriction enzymes were from Promega Biotech (Madison, WI, USA) or New England Biolabs (Beverly, MA, USA). Immunochemicals were obtained from Organon Technika (Malvern, PA, USA).

\section{Preparation and transfection of GFP-ensconsin con- structs}

Preparation of pEGFP-NI (Clontech Laboratories, Inc., Palo Alto, CA, USA) that encoded a GFPEMTB chimera, called pGFP-EMTB (including amino acids 18-272 of E-MAP-115 (ensconsin) followed by GFP) has been described elsewhere (11). Preparation of p5xGFP-EMTB, encoding a multimer of five GFP molecules (5xGFP) linked in tandem to the C-terminus of the MT-binding domain of ensconsin (EMTB), followed procedures and PCR conditions outlined in Faire et al. (11). For this construct, PCR utilized a 5 ' primer of sequence GGTACCGTCGACCCTGAGGGCTCTT CG (nt 924-949 of E-MAP-115), and a 3' primer of sequence GGTACCGAATTCGCGGCCGCTTTCCTTGT (nt 1361-1390 of GFP) to amplify the GFP-EMTB cassette from pGFP-EMTB. Note that the $3^{\prime}$ primer was modified in order to delete the stop codon of GFP, and that a Kpnl restriction enzyme site introduced into both primers was used to ligate the purified PCR product into p4XGFP-EMTB.

In order to verify that the additional GFP-encoding cassette had been inserted into the plasmid in the appropriate orientation and in the proper reading frame, we performed a ransient transfection, used FACS to isolate transfectants, and performed western blotting to verify that the molecular mass of the expressed protein ( $>205 \mathrm{kDa}$ ) was correct, before subcloning junctions and sequencing them according to Faire et al. (11).

Transfection of GFP-EMTB constructs into African green monkey kidney cells (TC-7 line) was described elsewhere (11). Transfection of pGFP-EMTB into HeLa cells followed the same protocol except that HeLa cells were transfected in their normal growth medium containing $10 \%$ calf, rather than fetal bovine, serum, and only $10 \mu \mathrm{L}$ of lipofectamine was incubated with cells and DNA, for a period of $16 \mathrm{hr}$, to obtain optimal transfection efficiency in this cell type.

Transient transfectants were either viewed in a fluorescence microscope $36-48 \mathrm{hr}$ after transfection to assess the efficacy of transfection and to monitor distribution of GFP chimeric protein, or they were sorted by FACS and placed in growth medium containing geneticin (G418) for at least $3 \mathrm{wk}$, to generate stable cell lines. G418-resistant clones of GFP-EMTB-HeLa cells were isolated using cloning cylinders (Bellco, Inc., Vineland, NJ, USA) and tested for their level of expression by immunofluorescence and western blotting; the expression level of these cells was constant for more than 40 passages in culture, either when cultured as monolayers in DMEM with $10 \%$ calf serum, or in suspension as previously described (1).

\section{Immunological analysis}

Guinea pig polyclonal antibodies raised against 6xhis-tagged-EMTB were used to detect GFP-EMTB chimeras on wescern blots processed as described previously (11), except that in some instances, as noted, blots were developed using enzyme-linked chemiluminescence (ECL). A 1:1000 dilution of guinea pig antihis-EMTB was used, and an ECL kit (Western Blotting Super Signal Substrate, Pierce Chemical Co., Rockford, IL, USA) was used to visualize immunoreactive bands, according to the manufacturer's instructions.

\section{Imaging of MTs in living transfected cells}

Images of cells grown on glass coverslips were acquired and processed as described in Waterman-Storer et al., (19) and F aire et al. (11). For 5xEMTB-GFP TC7 cells, and all other GFP-EMTB cell lines, a $1 \mathrm{sec}$ exposure time was used, to facilitate comparisons of relative brightness. 


\section{Isolation and assay of MTs from transfected cells}

GFP-EMTB-HeLa cells were cultured and processed to obtain extract, as described by Bulinski and Bossler (3). Extracts prepared in PDEMG buffer $(0.1 \mathrm{M}$ PIPES, pH 6.9, $1 \mathrm{mM}$ each of dithiothreitol, EGTA, and $\mathrm{MgCl}_{2}$, and GTP) containing $1 \mu \mathrm{g}$ each chymostatin, leupeptin, antipain, and pepstatin, were supplemented with $10 \mu M$ Taxol, the extract was warmed for 20 min to allow MT polymerization, and MTs were isolated by sedimentation $(40,000 \times \mathrm{g}, 30 \mathrm{~min})$ through a solution of $20 \%$ sucrose in PDEMG. The pellet, containing crude MTs, was washed $5 \mathrm{x}$ in 5 vol PDEMG buffer, and divided into six equal parts. Each was extracted, in parallel, with a different concentration of $\mathrm{NaCl}$, added to PDEMG buffer. Calcium depolymerization of crude MTs, to isolate calcium-sensitive and resistant fractions, was performed according to Bulinski and Bossler (3).

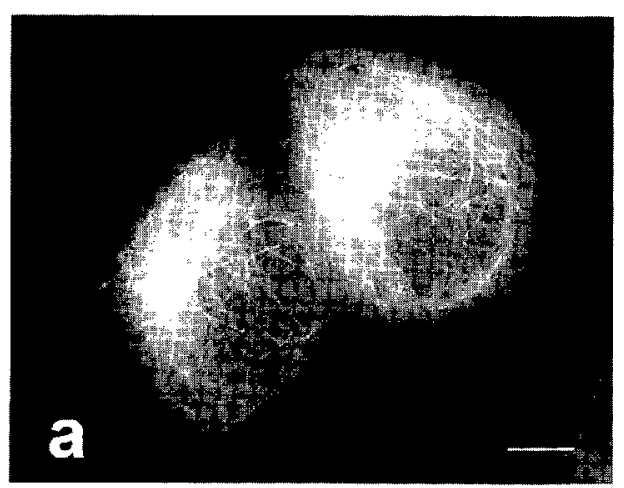

C

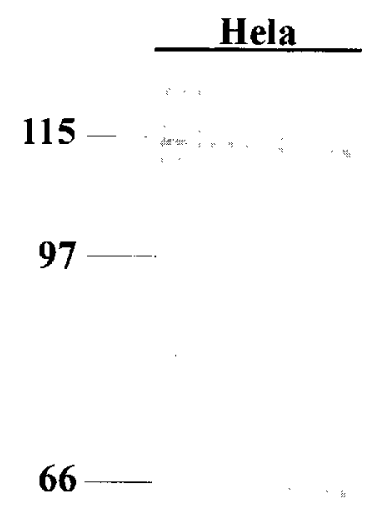

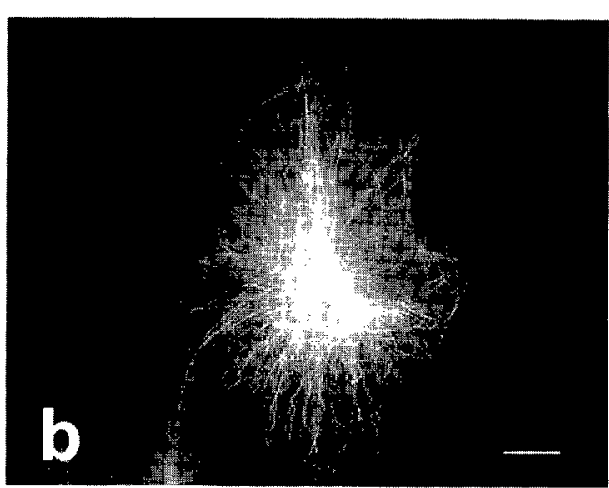

$\underline{\text { TC-7 }}$
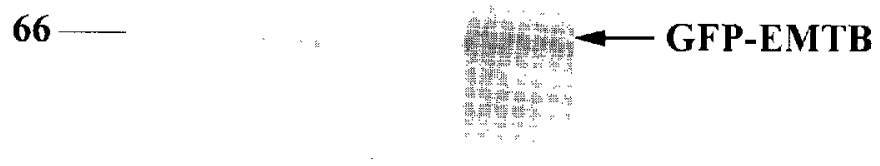

\section{2}

Fig. 1. Expression of Ensconsin Microtubule-Binding Domain (EMTB) in TC-7 and HeLa cells. (a, b) Micrographs of living, stably transfected HeLa and TC-7 cells, respectively, expressing a chimera, GFP-EMTB, consisting of a green fluorescent protein molecule linked to the MT-binding domain of ensconsin. Note that GFP-EMTB is distributed along cytoskeletal fibers; immunofluorescence (not shown) demonstrated that these fibers coincided with MTs. Bar in each panel represents $10 \mu \mathrm{m}$. (c) Western blot of 1, HeLa, 2, GFP-EMTB-HeLa, and 3, GFP-EMTB-TC-7 cell extract protein, immunostained with anti-EMTB antibody. Note that on this blot, in which immunoreactive bands were visualized with chromogenic chemical substrate, 4-chloro-1-napthol, both endogenous ensconsin and transfected GFP-EMTB can be detected in HeLa cells, while in TC-7 cells only the transfected protein can be detected, since ensconsin is not as abundant in the TC-7 cell line. $40 \mu \mathrm{g}$ of protein was loaded for each lane of the blot. 


\section{Results and Discussion}

\section{A GFP chimeric MAP domain is associated with microtubules in transfected cells}

In order to examine the distribution, dynamics, and function of E-MAP-115 (ensconsin) under in vivo conditions, we stably transfected African green monkey kidney cells, TC-7, and human cervical carcinoma cells, $\mathrm{HeLa}$, with plasmids encoding EMTB, the MT-binding domain of ensconsin, conjugated at its $\mathrm{C}$-terminus to GFP (GFP-EMTB; Fig. 1, a, b). We chose these two cell types because TC-7 cells are amenable to detailed morphological studies, owing to their flat morphology, while HeLa cells are biochemically tractable because they can be cultured in large quantities in suspension culture.

Figure 1 shows that GFP-EMTB was associated with MTs, as was endogenous ensconsin in the same cells (see for example, 3); no other fluorescent structures were observed. Judging from the fluorescence intensity of individual cells, the expression level exhibited amongst different cells in the population was somewhat heterogeneous, even after we had isolated clonal populations of cells. The western blot in Figure 1c shows the average content of GFP-EMTB within each population; content of GFP chimera was constant during more than ten (TC-7 cells) or as many as 40 (HeLa cells) passages in culture. No matter how much GFPEMTB the cells expressed, and therefore, how intense the fluorescence, comparisons with anti-tubulin immunofluorescence patterns always showed complete coincidence with the GFP-EMTB pattern. From these data we concluded that GFP-EMTB was associated with all MTs at all stages of the cell cycle, just as the endogenous MAP was (data not shown). Thus, in its steady-state intracellular distribution, GFP-EMTB appeared to be equivalent to the endogenous, full-length protein from which it was derived, suggesting that in vivo localization of ensconsin was determined independently of the absence of other MAP domains or the presence of appended GFP.

\section{Microtubule-binding properties of GFP chimera and endogenous MAP are equivalent}

Localization of GFP-EMTB along MTs in vivo showed qualitatively that the GFP chimeric molecule was capable of binding to the MT; however, it did not provide information about how avidly GFP-EMTB might be binding to MTs. Extraction with non-ionic detergent failed to extract GFP-EMTB from the cytoskeleton (11). Since analogous behavior had been reported previously for endogenous E-MAP-115 (ensconsin) $(3,15)$ detergent extraction results provided additional qualitative information that GFP-EMTB was bound to MTs as tenaciously as endogenous enscon- $\sin$.

To obtain quantitative information about the MTbinding properties of GFP-EMTB, we isolated Taxolstabilized MTs from extracts of GFP-EMTB-HeLa cells. As showr. in Figure 2A, a sedimentation assay of these MTs, ex racted with various concentrations of salt, showed that salt concentrations greater than $0.3 \mathrm{M}$ were required to elute either GFP-EMTB or endogenous ensconsin from the MTs. In addition, as quanti-

\section{A}

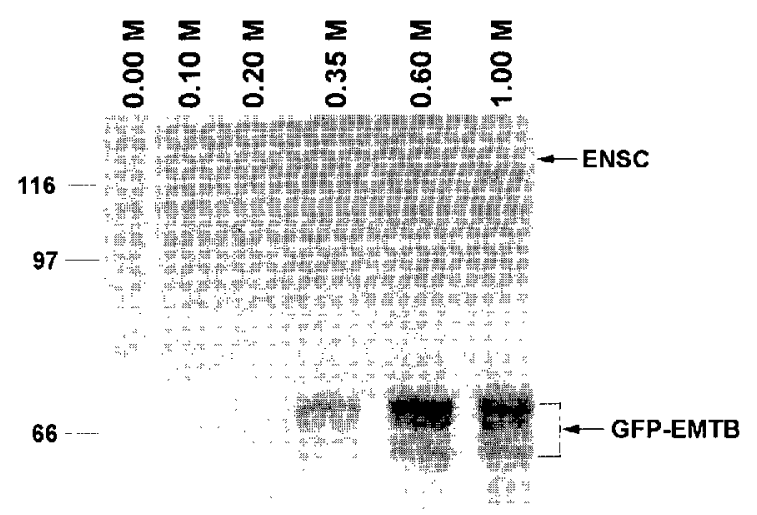

B

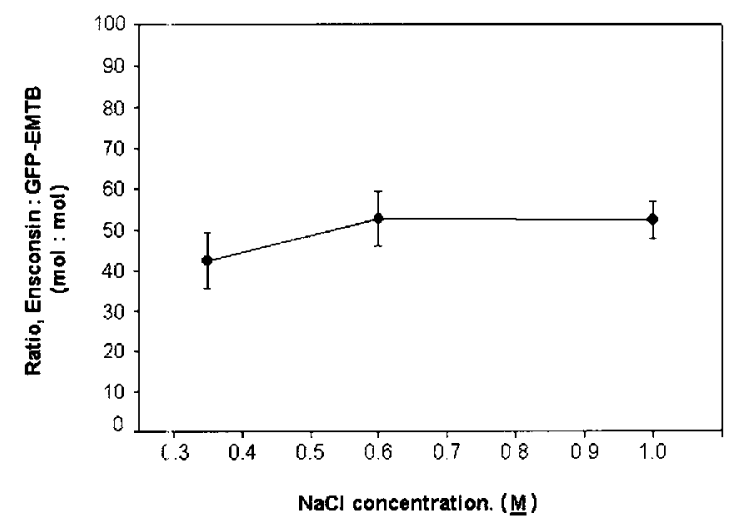

Fig. 2. GFP-EMTB and endogenous E-MAP-115 (ensconsin) bind equivalently to MTs. (A) MT samples prepared from GFP-EMTBHeLa cells were ex racted with different concentrations of salt, as described in the Materials \& Methods section. The western blot, immunostained with anti-EMTB antibody, shows that both endogenous ensconsin and GFP-EMTB eluted from the MTs only at salt concentrations greater than $0.3 M$. (B) Quantification of the ratio of endogenous MAP to GFP-EMTB eluted at each salt concentration used in extraction $€$ xperiments. Partitioning of endogenous MAP and GFP-EMTB was equivalent at each salt concentration used to elute MTs, suggesting that the two molecules bind equally tightly. Error bars represent standard deviation in the experiment $(\mathrm{N}=2)$. 
fied in Figure 2B, GFP-EMTB and endogenous ensconsin eluted identically from the salt-extracted MTs; the two molecules were present in equal ratio in the material eluted from MTs at each salt concentration. The fact that GFP-EMTB and endogenous ensconsin partitioned identically at each salt concentration suggested that both GFP-EMTB and endogenous ensconsin were bound to MTs with equivalent affinity.

As a further test of MT-binding properties, we partially depolymerized MTs from GFP-EMTB-HeLa cells by homogenizing them in ice-cold, calcium-containing buffer. Although calcium-induced proteolysis made quantification somewhat problematic, both GFPEMTB and endogenous ensconsin were highly enriched in that fraction of MTs that was calcium-stable (Fig. 3). Thus, both native and transfected MAP species appeared to be binding equally avidly to cellular MTs. The demonstration that behavior of GFP-EMTB chimera effectively mimics MT-binding properties of endogenous ensconsin supports the notion that ensconsin's MT-binding behavior is unaffected by either removing the projection domain, or attaching an exogenous GFP domain.

Our demonstration that GFP-EMTB and endogenous MAP show indistinguishable MT-binding proper-

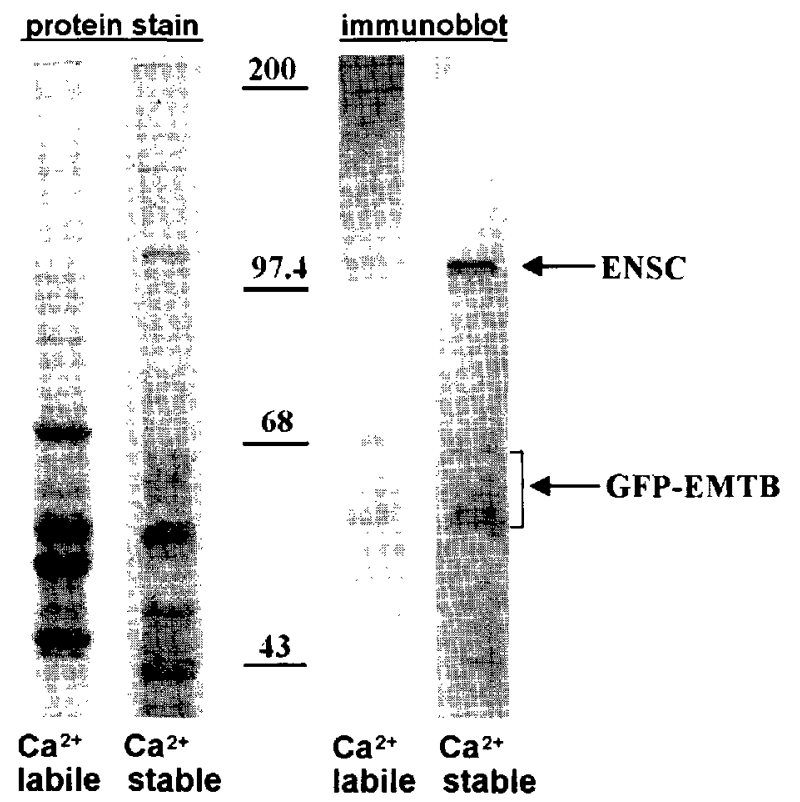

Fig. 3. Both GFP-EMTB and endogenous E-MAP-115 (ensconsin) associate with a $\mathrm{Ca}^{2+}$-stable subset of MTs. Taxol-stabilized MTs from GFP-EMTB-HeLa cells were fractionated into calcium-labile and calcium-stable subsets, as described in Bulinski and Bossler (1994); an electropherogram and a western blot immunostained with anti-EMTB is shown for each. Note that both endogenous ensconsin and GFP-EMTB are enriched relative to tubulin in the calcium-stable fraction. $40 \mu \mathrm{g}$ of protein was loaded for each lane of the blot. ties suggests that the GFP-EMTB chimera is an authentic reporter for the MAP's in vivo MT-binding behavior. Naturally, domains outside of the MT-binding domain, namely the proline-rich 'PAPA' or hinge region, and/or the projection domain at the molecule's C-terminus, are likely to exhibit functional interactions with molecules or systems outside of the MT (15). For example, the demonstration that a site that lies outside of the MAP's MT-binding domain undergoes phosphorylation at the onset of M-phase suggests that some MAP behavior or interaction is modified during the cell cycle (16).

As yet, we have no information about possible functions for MAP domains outside of the MT-binding domain; however, two results suggest that such functions will eventually be uncovered. First, wild-type TC-7 cells expressing a moderate level of GFP-EMTB chimera (a level four times greater than that endogenous to a HeLa cell) were not altered in MT dynamics, suggesting that this MAP does not function as an in vivo MT stabilizer (11). Since regulation of MT dynamics does not appear to be the raison d'être of the MT-binding domain of ensconsin, its binding to MTs may instead serve to tether molecules to the MT through their binding to the MAP's projection domain.

Second, an abnormal phenotype obtained when a dominant-negative construct is overexpressed often provides evidence for the function of proteins or their domains. One possible explanation for the finding that very high expression of GFP-EMTB in TC-7 cells was deleterious to cell growth and division (11) is that this single domain of ensconsin acted as a dominant negative whose expression inhibited functions of the MAP's projection domain.

Thus, the GFP-EMTB chimeric molecule serves as a useful and authentic reporter for the endogenous molecule in living cells, because its localization and binding mimic that of the intact, endogenous MAP. However, the MAP's projection domain undoubtedly displays functions whose characterization remains to be elucidated, and studies of GFP-MTB do not provide information about these functions.

\section{Generation of GFP-MAP chimeras with improved fluorescence characteristics}

Figure 1 shows that expression of GFP-EMTB provides sufficient fluorescence to allow detection of labeled MTs in live images of stable transfectants. However, we found that phenotypic abnormalities were exhibited by cells stably expressing very high levels of GFP-EMTB, as has also been noted for cells overexpressing other MAPs such as MAP4 $(17,18)$ and tau (7). Meanwhile, insufficient initial fluorescence intensity and considerable photobleaching of cells with lower level expression precluded many types of experi- 
ments (11). Since it was also reported that the fluorescence of individual GFP molecules was variable during long-term observation, due to a phenomenon called 'blinking' or 'switching' (6), we previously conjugated two, three or four GFP molecules in tandem to each EMTB moiety. Developing cell lines transfected with these allowed us to express lower levels of GFP-EMTB chimeras, while obtaining brighter, steadier fluorescence, and decreased impact from photobleaching (11).

Because of our initial success in appending multimers of GFP to EMTB, we decided to exploit this emerging technology, maximizing the number of GFP molecules that we could append to the MAP domain. An important technical goal was to improve the fluorescence intensity and stability without encountering insumountable problems, either in generating transfectants or in isolating cells that expressed reasonable levels of GFP-MAP chimera. In addition, in order to use GFP-
EMTB chimeras to examine MAP: MT dynamics by fluorescent speckle microscopy (20), it was desirable to append as many GFP molecules as feasible to a single EMTB molectle, in order that each resolvable speckle would contain as few molecules of EMTB as possible. Ultimately, pushing the system to its limit might even allow us to detect individual molecules of EMTB, expressed at low level, conjugated to many GFP moieties.

The western blot in Figure 4a documents the expression of 5xGFP-EMTB chimera in TC-7 cell transfectants. The low level of expression of 5xGFP-EMTB chimera in these cells was detectable only by ECL; by comparison with other cell lines, we estimate that between three and five molecules of 5xGFP-EMTB were present along each micrometer of the MTs in these cells (data not shown). Expression was somewhat heterogeneous and we have yet to isolate clonal populations of 5xGFP-EMTB-TC-7 cells; however, we found that cells

a
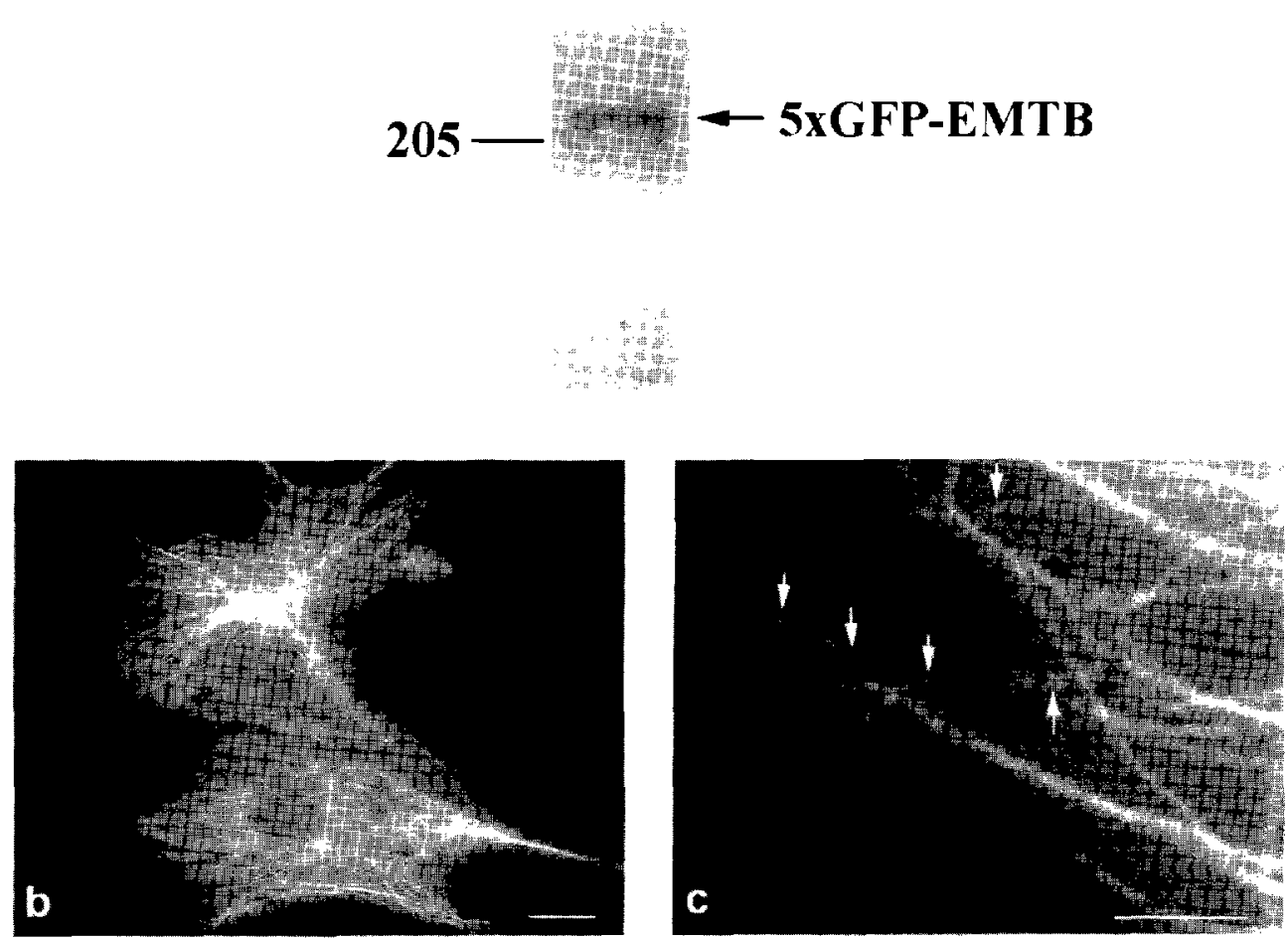

Fig. 4. Expression of 5xGFP-EMTB in TC-7 cells. (a) Western blot of total cell extracts of cells expressing five GFP molecules conjugated to EMTB (5xGFP-EMTB). Sample was prepared from 190,000 5xGFP-EMTB-TC-7 cells; after transient transfection, cells were sorted by FACS for highest expression. Blots were electrophoresed, stained with anti-his-EMTB, and developed with ECL, as described in Materials and Methods. The band corresponding to 5xGFP-EMTB is shown, as is the position of a molecular mass marker of $205 \mathrm{kDa}$. (c, d) Micrographs of TC7 cells expressing multimeric 5xGP-EMTB construct. Both low (b), and high (c) magnification micrographs of 5xGFP-EMTB-TC-7 cells are shown. Note that in (b) MTs can be seen to be labeled with GFP-MAP chimera, while in (c) discontinuities in the fluorescence intensity can be observed; these are labeled with arrows. These discontinuous bright spots represent speckles containing discrete molecules or groups of molecules of 5xGFP-EMTB, Quantification of the expression of 5xGFP-EMTB in these cells suggests that they contain 3-5 molecules of 5xGFPEMTB per micron of MT; thus, each resolvable speckle contains only one or a few molecules of GFP-MAP chimera. The bar in (b) indicates 10 $\mu \mathrm{m}$; the bar in (c) indicates $5 \mu \mathrm{m}$. 
were stable in their level of expression during many passages in culture.

As predicted, examination of 5xGFP-EMTB-TC-7 cells (Fig. 4b) showed that cells that expressed EMTB conjugated to multiple GFP molecules yielded levels of fluorescence requisite for extensive time-lapse imaging of single MTs. In addition, the 5xGFP-EMTB-TC-7 cells showed photobleaching effects that were less profound than we had previously observed in cells expressing the original $(1 \times)$ GFP-EMTB. We also noted that fluorescence intensity was quite steady during observation. Finally, as we had noted previously in $4 \mathrm{xGFP}$ EMTB-TC-7 cells (11), we found that fluorescence along single MTs in 5xGFP-EMTB-TC-7 cells gave a speckled appearance when viewed at high magnification (Fig. 4c). This property may be useful in future studies of the dynamics of association of ensconsin with MTs.

We have not attempted to construct GFP-MAP chimeras containing more than five GFP molecules appended to each EMTB molecule. For several reasons, generation of 5xGFP-EMTB may be a suitable endpoint for us; that is, appending five GFPs to a protein of interest may represent an upper limit for obtaining optimal protein labeling. As shown in Figure 4a, the molecular size of the 5xGFP-EMTB chimera is large. Electrophoresis yields an apparent $M_{r}>205 \mathrm{kDa}$, which is larger than the apparent $M_{r}$ of the endogenous fulllength ensconsin molecule ( $115 \mathrm{kDa})$. Although we have not observed any deleterious effects of expression of EMTB molecules containing five added GFPs, generating proteins of such large size and binding them to cellular MTs might begin to impact negatively upon MT functions or interactions.

A second reason that five appeared to constitute an upper limit for the number of GFPs that could be used in protein labeling is that efficiency of transfection and level of expression both decrease as the transfected constructs become larger. For example, because transfection efficiency is low, isolation of permanent cell lines expressing either $4 \mathrm{x}$ - or 5xGFP-EMTB required presorting by FACS; transfectants were not prevalent enough to allow us to isolate expressing colonies with drug selection alone. We also found that addition of each GFP to the EMTB construct reduced the level of protein expression by $25-60 \%$ (e.g., see Table III, 11 ). Were we to add more than five GFPs, we predict that protein expression might prove to be so low in level that it might be difficult to detect by western blot, even with sensitive ECL reagents. Most important, expression of 5xGFP-EMTB in TC-7 cells, estimated at only 3-5 molecules of GFP-MAP chimera per micron of MT (Fig. 4a), was a level sufficiently low to allow us to detect single MAP molecules, a major goal in constructing the multimeric GFP chimeras.
Finally, we note that two previous improvements in GFP-tagging (13), for example, those were achieved via mutation of the wild type GFP from Aequorea victoria (4) at amino acid positions S65T (7) and F64L (5), each rendered fluorescence intensity that was brighter by a factor of 5-6. Ironically, conjugating our target protein, the MAP domain, EMTB, to five GFPs instead of one also increased fluorescence intensity yield per molecule by a factor of five. Thus, appending five GFP molecules to a single EMTB molecule has created an improvement in brightness as significant as each of the past genetic mutations. Using $5 \mathrm{xGFP}$ as a protein label has thus allowed us to obtain bright fluorescence without requiring a deleteriously large amount of GFPchimera expression. GFP multimers have concomitantly mutigated effects of photobleaching and concerns about blinking/switching phenomena during long-term imaging (6).

Acknowledgments. We wish to thank Drs. Ted Salmon and Clare Waterman-Storer for discussions that motivated improvements in labeling using multiple GFP constructs. We also thank Drs. Michael Sheetz and David Odde for invigorating discussions, and Ms. Aparna Prasad for technical assistance. Support from a National Institutes of Health Grant to JCB (CA 70951), a Summer Undergraduate Research Fellowship (SURF) from Columbia University to PP, a postdoctoral fellowship from NIH to KF (AR 08316), and an NIH predoctoral traineeship to WC (GM 08224), are also gratefully acknowledged.

\section{References}

1. BULINSKI, J.C. and BoRisy, G.G. 1979. Self-assembly of Hela tubulin and the identification of HeLa microtubule-associated proteins. Proc. Natl. Acad. Sci. USA, 76: 293-297.

2. Bulinski, J.C. and BoRISY, G.G. 1980. Microtubule-associated proteins from cultured HeLa cells: analysis of molecular properties and effects on microtubule polymerization. $J$, Biol. Chern., 255: 11570-11576.

3. Bulinski, J.C. and Bossler, A. 1994. Purification and characterization of ensconsin, a novel microtubule stabilizing protein. J. Cell Science, 107: 2839-2849.

4. Chalfie, M., Tu, Y., Euskirchen, G., Ward, W.W., and Prasher, D.C. 1994. Green fluorescent protein as a marker for gene expression. Science, 263: 802-805.

5. CoRmack, B.P., Valdivia, R.H., and Falkow, S. 1996. FACS-optimized mutants of the green fluorescent protein (GFP). Gene, 173: 33-38.

6. Dickson, R.M., Cubitt, A.B., TSIEN, R.Y., and MoErner, W.E. 1997. On/off blinking and switching behaviour of single molecules of green fluorescent protein. Nature, 388: 355-358.

7. Ebneth, A., Godemann, R., Stamer, K., Illenberger, S., TrinczeK, B., and MaNDELKow E. 1998. Overexpression of tau protein inhibits kinesin-dependent trafficking of vesicles, mitochondria, and endoplasmic reticulum: implications for Alzheimer's disease. J. Cell Biol., 143: 777-794.

8. Ehrig, T., O'Kane, D.J., and Prendergast, F.G. 1995. Green fluorescent protein mutants with altered excitation spectra. FEBS Letters, 367: 163-166. 
9. Fabrí-Jonca, N., Allaman, J.-M., Radigruber, G., Meda, P., Kiss, J.Z., French, L.E., and Masson, D. 1998. The distribution of murine 115 -kDa epithelial MT-associated protein (E-MAP-115) during embryogenesis and in adult organs suggests a role in epithelial polarization and differentiation. Differentiation, 63: 169-180.

10. Fabré-Jonca, N., Viard, I., French, L.E., and Masson, D. 1999. Upregulation and redistribution of E-MAP-115 (epithelial microtubule-associated protein of $115-\mathrm{kDa}$ ) in terminally differentiating kerarinocytes is coincident with the formation of intercellular contacts. J. Invest. Dermatol., 112: 216-225.

11. Faire, K., Waterman-Storer, C., Gruber, D., Masson, D., SAlMON, E., and Bulinski, J.C. 1999. Dynamic in vivo behavior of GFP-labeled E-MAP-115 (ensconsin) in cultured cells. $J$. Cell Sci., in press.

12. Gruber, D., Chang, W., Faire, K., and Bulinski, J.C. 1999. Expression and binding of the microtubule-associated protein, E-MAP-115 (ensconsin), in Taxol-treated cells. Manuscript submitted.

13. Heim, R. and Tsien, R.Y. 1996. Engineering green fluorescent protein for improved brightness, longer wavelengths and fluorescence resonance energy transfer. Curr. Biol., 6: 178-182.

14. Mandelkow, E. and MandelKow, E.M. 1995. Microtubules and microtubule-associated proteins. Curr. Opin. Cell Biol., 7: $72-81$.
15. MAsson, D. and Kreis, T.E. 1993. Identification and molecular characterization of E-MAP-115, a novel microtubule-associated protein predominantly expressed in epithelial cells. $J$. Cell Biol., 123: 357-371.

16. MAsson, D. and KREIs, T.E. 1995. Binding of E-MAP-115 to microtubules is regulated by cell cycle dependent phosphorylation. J. Cell Biol., 131: 1015-1024.

17. Nguyen, H.-L., Chari, S., Gruber, D., Lue, C.-M., Chapin, S.J., and BuLinSKı, J.C. 1997. Overexpression of full- or partial-length MAP4 stabilizes microtubules and alters cell growth. J. Cell Sci., 110: 281-294.

18. Olsen, K.R., MClntosh, J.R., and Olmsted, J.B. 1995. Analysis of MAP4 function in living cells using green fluorescent protein (GFP) chimeras. J. Cell Biochem., 130: 639-650.

19. Waterman-STORER, C.M. and Salmon, E.D. 1997. Actomyosin-based retrograde flow of microtubules in the lamella of migrating epithelial cells influences microtubule dynamic instability and turnover and is associated with microtubule breakage and treadmilling. I. Cell Biol., 139: 417-434.

20. Waterman-Storer, C.M., Desai, A., Bulinski, J.C., and SALMON, E.D. 1998. Fluorescent speckle imaging: visualizing the movement, assembly, and turnover of macromolecular assemblies in living cells. Current Biology, 8: 227-230.

21. Weatherbee, J.A., Luftig, R.B., and Weihing, R.R. 1980. Purification and reconstitution of HeLa microtubules. Biochemistry, 19: 4116-4123. 